\title{
Pre-Analysis Plan \\ Persuasion in Medicine: \\ Experimental Evidence on Sender and Signal Effects
}

\author{
This Draft: October 12, 2020
}

\section{Introduction}

The aim of this study is to identify which sender/signal combinations are most persuasive in encouraging low socioeconomic males living in the United States to take up seasonal flu vaccination. We plan to recruit subjects and randomly assign them to persuasion treatments in the form of video infomercials that vary dimensions of the sender of the medical recommendation (race and gender concordance, "expert," and empathy treatments). Specifically, we will cross-randomize race with the "expert" treatment as the actor in the video will portray either a doctor or a layperson. In addition, we may randomize gender of the actor for subjects assigned to a race-concordant "expert" sender, given sufficient funds. The script used in the experiment will be varied between one that acknowledges past injustices and one that does not. ${ }^{1}$ Our outcomes of interest are posterior beliefs about seasonal flu vaccination, WTP for a free flu shot coupon and redemption of the coupon.

We are interested in testing the following hypotheses: (1.a) whether low-income male subjects (both pooled and separately by race) are more likely to be persuaded by race-concordant doctors; (1.b) whether the subjects assigned to a race-concordant doctor are more likely to update/change their beliefs/behavior by gender-discordant senders; and, (2) whether the racial concordance effect is similar for Black and white male respondents. In addition, given the pipeline issues associated with increasing the supply of Black male physicians in the short-run, a policy relevant question is whether community health workers (CHW) or other ancillary staff could function in place of medical doctors when recommending or informing about preventive health services. This could be a particularly effective strategy for distributing routine preventive medical care. ${ }^{2}$ Theoretically, doctors may have more expertise when it comes to medical issues and may be viewed as more trustworthy, but laypeople might be socially more proximate to the men we are trying to reach. Thus, we will test, within Black respondents, whether a Black male doctor has the same effect as a Black male layperson (Hypothesis (3)). Finally, for similar (pipeline) reasons, we are interested in whether the current stock of medical doctors (which has relatively few African-American and Hispanic doctors) could be trained in such a way to bridge any racial discordance effect (if one is shown to exist in our context). Thus, among Black respondents, we

\footnotetext{
${ }^{1}$ See Section 3.3 for transcripts of the scripts.

${ }^{2}$ The CHW model has been used extensively in developing world settings and for prenatal care in the U.S. See Srinivas et al (2019).
} 
will randomize them to white doctors that give a standard script vs. those that provide a more empathetic signal ${ }^{3}$, and we will test, within Black respondents, whether the white doctor empathy treatment has the same effect as the white doctor standard treatment (Hypothesis (4)).

The basic design requires collection of baseline and endline surveys combined with administrative data from pharmacies about coupon redemption. The outcomes of interest are posterior beliefs about the safety of seasonal flu vaccination, WTP for a free flu shot coupon, and redemption of the coupon.

Our field experiment has the following structure:

1. Recruitment and Baseline Survey

(a) Recruit via Qualtrics during the flu season. ${ }^{4}$

(b) Collect demographic information and healthcare experience.

(c) Elicit prior likelihood/intent for getting vaccinated and probability of getting sick with the flu.

(d) Elicit priors from subjects about flu shot safety.

(e) Assign video treatment.

(f) Elicit willingness-to-pay (WTP) for a flu shot coupon with a multiple price list.

(g) Elicit posterior likelihood/intent for getting vaccinated.

(h) Elicit posteriors about flu shot safety .

(i) Elicit social distance between the sender and receiver.

(j) Elicit ratings on the sender in the video.

(k) Elicit attention to signal in the video.

(l) Distribute flu shot coupons by email.

2. Endline Survey (2 weeks after baseline) ${ }^{5}$

(a) Elicit posteriors about flu shot safety.

(b) Elicit self-report on whether received flu shot.

(c) Elicit recall of information from baseline video.

(d) Debrief subjects.

3. Between Baseline and Endline

(a) Collect information on flu shot coupon redemption from corporate partner.

\footnotetext{
${ }^{3}$ Although we could test both the layman and empathy with white respondents as well, it's not clear how white respondents will interpret the signal. Prior piloting showed that white respondents assumed that Black respondents dressed as laymen were less educated, which might make them more socially proximate than white men dressed as laymen. In addition, by focusing our hypotheses on policy-relevant parameters, we increase our power by reducing the number of arms from 16 to 6.

${ }^{4}$ In a pilot, we also experimented recruiting via online ads on Facebook and Google, but discontinued this path of recruitment due to budget concerns and insufficient targeting capabilities.

${ }^{5}$ We sent out the endline survey 3 months after baseline in a pilot, but have shifted the release of the endline earlier to reduce attrition.
} 


\section{Hypotheses Tested}

In this study, we seek to answer the following research questions:

1. (a) Does racial concordance between an expert (i.e. doctor) sender and receiver affect flu shot beliefs and/or behavior, and (b) does a subject update beliefs more or change behavior with a gender-discordant expert in a racial concordance setting? ${ }^{6}$

2. Is the effect of race concordance between expert sender and receiver stronger for Black vs. white men?

3. Does a layperson sender work as well as an expert figure (i.e. medical doctor) in shifting the beliefs and/or behavior of Black respondents?

4. If negative effects of a race-discordant expert sender to receiver exist, can these be mitigated by a signal that acknowledges past injustices?

\section{Research Strategy}

\subsection{Recruitment and Sampling}

We will recruit for this study using Qualtrics. Our target population includes adult US males with a high school degree or less, who have not yet received the flu vaccine in the current flu season. We aim to recruit 70\% non-Hispanic Blacks and 30\% non-Hispanic Whites.

The target population is defined by the following criteria:

Demographic criteria For inclusion in our study are the following:

- Age: between 30 and 50 years old.

- Education: High school education or less.

- Gender: Male.

- Race: non-Hispanic Black or non-Hispanic white.

- Has not received the flu shot in the current flu season yet.

- Has sound on his device turned on.

Data quality checks Among the subjects who complete the baseline survey, we will further exclude subjects from the analysis if they satisfy the following indicator for low-quality survey responses:

- Fast survey-taking: Within race and treatment group, among the 5\% fastest in terms of total time spent on survey.

\footnotetext{
${ }^{6}$ We will test Hypothesis 1(b) funds permitting.
} 
- Nonsense answers in the open text sections that suggest low effort. ${ }^{7}$

- Inconsistent responses to questions. ${ }^{8}$

- Repeat survey takers. ${ }^{9}$

Flu shot coupon assignment Non-zero price draw: Among the subjects who complete the baseline survey, we will further exclude subjects from the analysis if they are offered the chance to trade-in their flu shot coupon for a cash reward in the WTP elicitation module. ${ }^{10}$

\subsection{Statistical Power}

In the power calculation below, we state minimum sample sizes needed in order to detect effect sizes of 0.2 in terms of standard deviation units of 10 main outcome variables ${ }^{11}$. Details on how we computed statistical power can be found in this document.

For hypothesis 1(a), we need 385 subjects for each treatment (i.e. racial concordance) and control (i.e. racial discordance) to test an overall racial concordance effect across both Black and white respondents assigned to an expert figure, detecting a difference in means (in standard deviation units) of 0.2. To detect this effect size within each racial group, we need 415 subjects for treatment (i.e. racial concordance) and control (i.e. racial discordance) group within Black respondents, and 370 subjects for treatment (i.e. racial concordance) and control (i.e. racial discordance) group within white respondents.

For hypothesis 1(b), we need 430 subjects for treatment (i.e. concordance) and control (i.e. gender discordance) group within Black respondents assigned to a race-concordant expert delivering a standard message. We need 450 subjects for treatment (i.e. gender concordance) and control (i.e. gender discordance) group within white respondents assigned to a race-concordant expert delivering a standard message.

For hypothesis 2, we need to double the sample size needed to test an overall racial concordance effect across both races assigned to an expert figure in order to test an interaction effect (i.e testing whether the effect of concordance varies by race of respondent).

\footnotetext{
${ }^{7}$ We refer to nonsense answers in the open text sections as answers that do not have decipherable English. We plan to have Mturkers code this.

${ }^{8}$ Examples of inconsistent responses are (1) a respondent responds that he earns a high income but is unemployed or on disability; (2) a respondent who replies inconsistently on WTP questions (i.e. he is willing to accept $\$ 1$ for trading in a flu shot coupon but not \$10).

${ }^{9}$ We will identify repeat survey takers by checking for duplicate email or IP addresses in survey data.

${ }^{10}$ The probability of receiving the coupon trade-in opportunity is $\frac{1}{1000}$.

${ }^{11}$ The 10 main outcome variables are: (1) difference between prior and posterior beliefs in flu shot side effects, (2) difference between posterior intent and prior intent for flu shot, (3) WTP price, (4) whether R wanted the link to look up pharmacy to redeem the coupon, (5) R's likelihood of recommending a flu shot to his friends/family, (6) R's likelihood of recommending the flu shot video to his friends/family, (7) R's subjective trustworthiness on the person in the video, (8) R's subjective rating on the qualification of the person in the video, (9) whether $\mathrm{R}$ would want to ask the person in the video about other health issues, and (10) whether person like the one in the video would be in R's contact.
} 
For hypothesis 3, we need 395 subjects for each treatment (i.e. Black respondents assigned to a raceconcordant expert delivering a standard message) and control group (i.e. Black respondents assigned to a race-concordant layperson delivering a standard message) to detect a 0.2 effect size in units of standard deviations.

For hypothesis 4, we need 430 for each treatment (i.e. empathetic treatment arm) and control (i.e. standard message arm) within Black respondents assigned to a race-discordant expert in order to detect a 0.2 effect size in units of standard deviations.

In conclusion, we need a sample size of 2,620 (1,720 Black and 900 white respondents) to test our main hypotheses described in section 5.4 with $80 \%$ power and at a significance level of 0.05 .

\subsection{Treatment}

We will randomize subjects across four different treatments, with respect to variation in the sender and message. Each treatment will involve an infomercial video in which a male actor gives information about the safety and efficacy of the flu shot. We will experimentally vary the: 1) race of the sender (either African American or Caucasian), 2) the sender "expert" (either doctor or layperson), and 3) the message (either M1 or M2). Funds permitting, we may also 4) vary the gender of the sender (either male or female) for the subjects assigned to a race-concordant sender.

We produced videos with a total of 5 African American and 5 Caucasian actors. All actors wear the exact same clothes, and they record the video four times representing the experimental variation discussed above. All actors were recruited from the same casting agency and have separately been rated on attractiveness and other characteristics via Mturk. We also collected data from imdb.com and mandy.com about age, education, height, and weight. In the doctor role, the actors wear a button-down blue shirt, striped tie, a lab coat and stethoscope. In the layperson role, they wear a white T-shirt. The baseline script video is about 40 seconds long and is reproduced below. ${ }^{12}$

Message 1 (M1) script reads:

The Centers for Disease Control and Prevention, or CDC, recommends everyone 6 months and older get the flu shot. The shot protects you from getting sick by cutting your chance of catching the flu in half. It's also very safe: less than 1 in 100 vaccinated people experiences a side effect such as fever or chills. The flu shot does not contain an active flu virus, so you cannot get the flu virus from the shot. I get the flu shot every year to protect myself, my family, and my community. I recommend you look into getting vaccinated as soon as possible.

Message 2 (M2) script is identical to the above, except that we added three more sentences acknowledging historical injustices committed by the medical establishment. They are highlighted in bold-face below:

\footnotetext{
${ }^{12}$ See appendix materials to this pre-analysis plan for two examples of the videos we recorded.
} 
The Centers for Disease Control and Prevention, or CDC, recommends everyone 6 months and older get the flu shot. I know some people are nervous to follow medical advice about vaccines. In the past, there may have been times when the medical community broke your trust. But I hope that sharing some information with you can help you understand how important the flu shot is. The shot protects you from getting sick by cutting your chance of catching the flu in half. It's also very safe: less than 1 in 100 vaccinated people experiences a side effect such as fever or chills. The flu shot does not contain an active flu virus, so you cannot get the flu virus from the shot. I get the flu shot every year to protect myself, my family, and my community. I recommend you look into getting vaccinated as soon as possible. ${ }^{13}$

We include as a screening question the capability to turn the sound on so individuals can hear the video.

\subsection{Assignment to Treatment}

Subjects will be randomly assigned to one of eight main treatment groups that differ in the four dimensions described in Section 3.3, with respect to variation in the sender and message. There will be an equal draw for Black and white senders, an equal draw for male and female senders within each race concordance group, an equal draw of M1 and M2, and an equal draw towards a expert vs. layperson. Within each treatment group except the gender treatment group, subjects will be randomly assigned to one of the 5 recorded actors of the assigned race. For the gender treatment group, subjects will be randomly assigned to one of the 10 recorded female or male race-concordant actors; we will assign subjects to each actor with equal proportion.

All subjects will receive a free flu shot coupon, with all individuals informed during WTP elicitation that they may have the opportunity to trade-in the coupon for a cash reward. Participants will be provided the flu shot coupon without the opportunity to trade it in for cash with probability $\frac{999}{1000}$. This is so that we can measure coupon redemption in the majority of the sample.

We checked whether asking priors changes behaviors via a "doubling down effect" or engaging System II vs. System I in decision-making. We did not see a strong effect and scaled up the asking of priors to all respondents (See Appendix Section D for pilot lessons).

Please see Figure 1 for the overview of the study design.

\footnotetext{
${ }^{13}$ For the layperson video, we replaced the word "cannot" with "can' $t$ " for the phrase "cannot get the flu virus from the shot."
} 
Figure 1: Study Design

Receiver Variation

$\checkmark \quad$ Sender Variation 1

Sender Variation 2

Signal Variation

Sender Variation 3

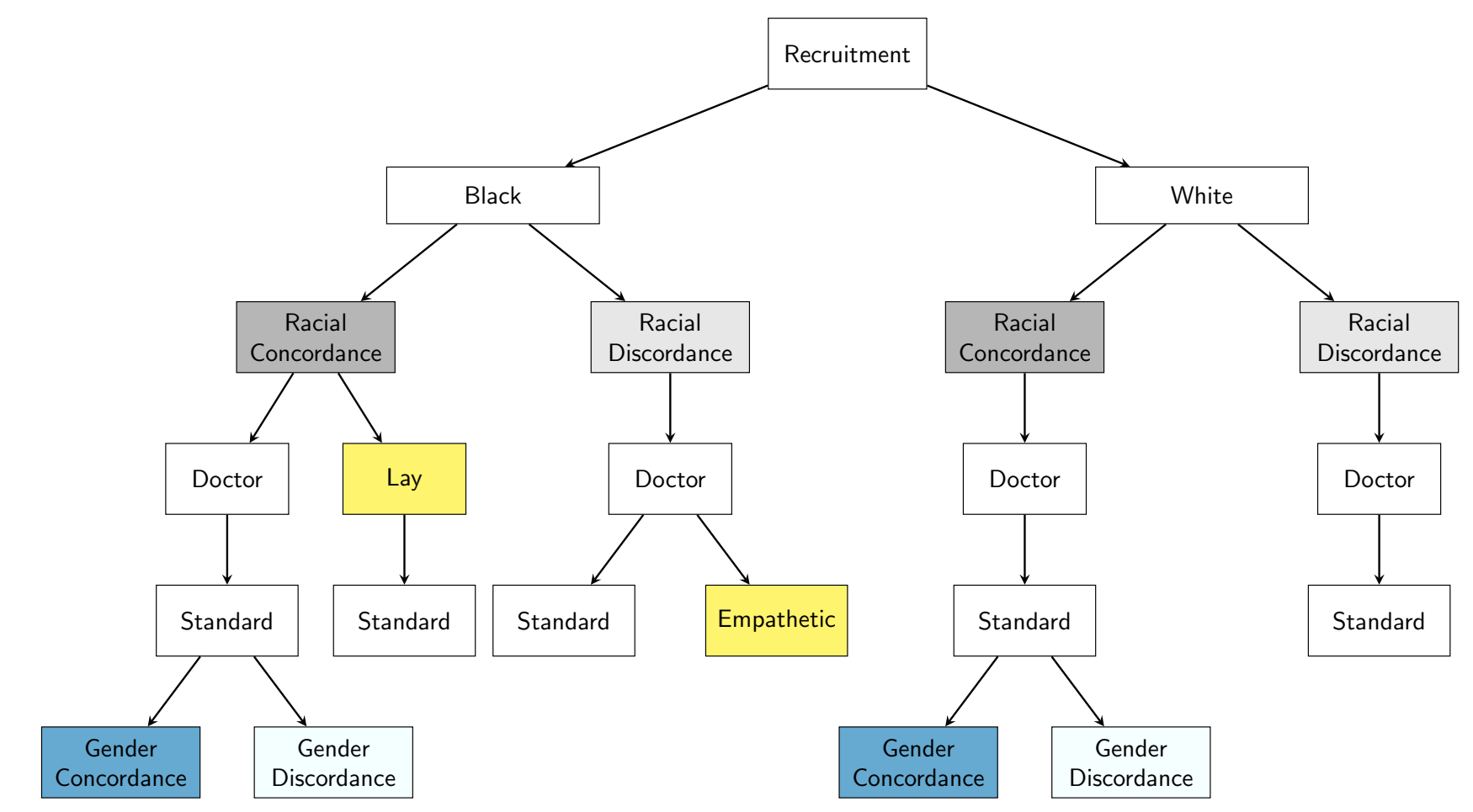




\subsection{WTP elicitation}

Redemption rates of the flu shot coupon (Redemption) as well as willingness to pay (WTP) for the coupon are the main outcomes of interest in this study. We will use the following method to elicit flu shot coupon valuations in an incentivized manner.

Immediately after the video treatment stage, we will offer the flu shot coupon to all participants. We will then inform participants that they may be offered the chance to trade in their flu shot coupon for cash. Participants will then be shown a multiple price list and asked, for each amount, whether they would prefer to keep the flu shot coupon or receive a cash reward of the given amount instead. ${ }^{14}$ The price list includes prices $\$ 1, \$ 2, \$ 5$, and $\$ 10$. Prices will be presented as a take-it-or-leave-it offers.

Following WTP elicitation, the computer will randomly choose at random whether the participant will be offered the chance to trade in the flu shot coupon for cash. If the participant is selected for a chance to trade in the coupon, the computer will proceed to randomly draw one price offer for that participant. If the subject accepted at the price drawn, he will receive the cash reward of the given amount in lieu of the flu shot coupon. If the subject rejected the price, he will not receive the coupon and keep the flu shot coupon instead. We will skew towards providing subjects with the flu shot coupon without the opportunity to trade it in so that we can observe redemption decisions for most subjects.

Since our target population is expected to have lower literacy and numeracy then other experimental samples, we have striven to keep our questions about WTP straightforward and provide an explanation in words of the consequences of each decision. ${ }^{15}$

\subsection{Coupon Redemption}

Our initial pilot revealed a very low take-up of the flu shot coupon. We have endeavored to improve take-up using two strategies. The first is to inform respondents that they can enter a lottery for $\$ 100$ if they redeem the coupon. The second is to make entry into the lottery contingent on redemption in a narrow window of time after coupon receipt so as to militate against procrastination.

This strategy is helpful under the following circumstance: Although our intervention shifts beliefs in the right direction (i.e. towards increased net benefits of the flu shot), beliefs about net benefits are still below the internal "threshold" for taking the action to redeem the coupon - see theories of behavior change in Prochaska and Wayne (1997). ${ }^{16}$ That is, even after our intervention subjects are potentially

\footnotetext{
${ }^{14}$ In a pilot, we paid participants $\$ 10$ for completing the baseline survey and elicited WTP by asking how much participants would be willing to deduct from their overall survey earnings to receive the flu shot coupon. For our main study, we are using Qualtrics to handle participant payment and deducting from earnings is not logistically feasible, particularly as participants will be paid less than $\$ 10$ and we do not have mechanisms in place to charge participants for the coupon.

${ }^{15}$ We also wanted to keep the survey length manageable and hence we do not have training/understanding questions in the survey.

${ }^{16}$ According to the transtheoretical model in Prochaska and Wayne (1997), behavioral change is a "process involving progress through a series of stages", which is composed of precontemplation, contemplation, preparation, action, maintenance, and termination.
} 
still in the contemplation phase. Our supposition is that the lottery will help clear the threshold for some, while preserving differences between the treatment groups that are due to treatment. ${ }^{17}$

\subsection{Attrition from the Sample}

The vast majority of our outcome variables will be collected in the baseline survey, and thus will not be subject to attrition. We expect few participants to drop out after watching the video but prior to completing the survey as they would forfeit payment. Secondary outcome variables collected in the endline survey may be impacted by attrition. These variables are "Flu shot - self report / spouse / child", all secondary outcomes in the "beliefs about the flu and flu shot" section, and all attention/recall questions elicited at endline.

\section{Fieldwork}

\subsection{Instruments}

We will collect data from two sources - online surveys and administrative data on flu coupon redemption from the pharmacies. The surveys will be hosted by the survey platform Qualtrics. Individual-level data on flu shot coupon redemption will be sent by TotalWellness, the company that will provide the flu shot coupons to us. The coupons are redeemable at all major pharmacies, such as CVS, Walgreens, Kroger, and Wal-Mart, in the US. ${ }^{18}$

\subsection{Incentives}

For the main study, Qualtrics will be responsible for administering payment for completing the baseline survey. ${ }^{19}$ Baseline survey payment will vary depending on the panel to which the participant belongs, but will average about $\$ 2.5$. Participants will receive a $\$ 5$ gift card for completing the endline survey. Among baseline survey participants who redeem the flu shot coupon within 5 days of coupon receipt, we will draw a lottery winner and pay the winner a $\$ 100$ gift card.

\subsection{Data Collection}

The field experiment will take place during the flu seasons of 2019-20 and 2020-21. Our piloting process commenced in October 2019 and ended in March 2020. ${ }^{20}$ Our main study will launch in October 2020,

\footnotetext{
${ }^{17}$ The one potential drawback is that financial incentive/lottery overpowers the lighter touch treatment that we give via the videos. Because we are not interested in whether incentives change behavior, we are not randomizing the lottery.

${ }^{18}$ See list of participating pharmacies here: https ://www. totalwellnesshealth. com/pharmacy-2/.

${ }^{19}$ Participants received a $\$ 10$ gift card for completing the baseline survey in our pilot, and a payment varying between $\$ 5$ and $\$ 10$ for completing the endline survey. We are planning to have Qualtrics administer the payment for budgetary and logistical reasons.

${ }^{20}$ We ran a total of two pilots. The first, which ran from October to December 2019, recruited using Qualtrics and was unsuccessful due to imprecise targeting of low-education individuals and too many (16) treatment arms. The second, which ran from December 2019 to March 2020, recruited using Qualtrics and online ads on Facebook and Google. We accrued 853 completed responses through Qualtrics. Our recruitment via online ads was unsuccessful, with an extremely low number of completed responses and high costs. Learning from the experiences of the pilots, we plan on scaling-up the Qualtrics
} 
with data collection projected to be completed by the end of the year.

Data collection will entail three components: baseline survey responses, endline survey responses, and data on flu shot coupon redemption. We will link the data across the three components using a unique numeric study identifier (ID) for each subject. In practice, we will assign the ID in the baseline survey, where we also collect subjects' email addresses. After a participant who is eligible for the coupon completes the baseline survey, he will receive the coupon immediately by email. The coupon has the subject's study ID printed on it. When a coupon is redeemed at a pharmacy, the pharmacist will record the ID and automatically pass it on electronically to TotalWellness for billing. TotalWellness in turn will collect the IDs of redeemed flu shot coupons and pass the list on to the research team for billing.

In addition to the above, we will collect data from a separate sample of low-income men regarding feedback on the actors including their attractiveness, tone and trustworthiness.

\subsection{Data Processing}

Survey responses collected on Qualtrics will be downloaded as a .csv file, and then cleaned and analyzed in Stata. Data on flu shot coupon redemption will be merged with the unique study ID of each subject. Any PII will be dropped from the dataset, since it is not needed for the analysis. It is anticipated that do files that create and analyze the data will be made available to other research teams. The clean data (without identifiers) will also be made available. Only non-PII information on the actors will be released.

\section{Empirical Analysis}

\subsection{Outcome Variable Construction}

\subsubsection{Flu shot take-up}

Primary outcomes:

- Flu shot coupon redemption: Indicator for whether a flu shot coupon was redeemed. Coded 1 if yes, and 0 if no.

Secondary outcomes:

- Flu shot likelihood - self: Collected before and after display of the video at baseline. Measured by answer to the survey question "How likely are you to get a flu shot before February 2020? - scaled from 0 (Not at all likely) to 10 (Extremely likely).

- Flu shot likelihood - self: Collected at endline. Measured by answer to the survey question "How likely are you to get a flu shot next flu season? - scaled from 0 (Not at all likely) to 10 (Extremely likely).

- Likelihood of recommending the flu shot to friends or family - self: Collected after display of the video at baseline. Measured by answer to the survey question "How likely are you to recommend the flu shot to a family member or friend? - scaled from 0 (Not at all likely) to 10 (Extremely likely).

survey from the second pilot with an additional 2,500 responses, for a total sample of about 3,350 individuals. 
- Flu shot - self report: Collected at endline. Indicator based on answer to the survey question Did you get the flu shot since you completed our first survey (i.e. since October 2019)? - Yes/No.

- Flu shot - spouse: Collected at endline. Based on answer to the survey question Did your spouse get a flu shot this season? - Yes/No/Don't have a spouse/Don't know. - Coded 1 if "Yes", 0.5 if "Don't know", 0 if "No", and missing if "Don't have a spouse".

- Flu shot - child: Collected at endline. Based on answer to the survey question Did your children get a flu shot this season? - Yes/No/Don't have children/Don't know. - Coded 1 if "Yes", 0.5 if "Don't know", 0 if "No", and missing if "Don't have children".

- Flu shot - household: Collected at endline. Based on answer to the survey question Did anyone in your household get a flu shot this season? - Yes/No/Live alone/Don't know. - Coded 1 if "Yes", 0.5 if "Don't know", 0 if "No", and missing if "Live alone".

Index of flu shot take-up:

- Includes all primary and secondary outcomes listed above that are measured at baseline. ${ }^{21}$

\subsubsection{Flu Shot Valuation (WTP)}

- Flu shot coupon WTP: Collected after display of the video at baseline. Measured using the WTP elicitation as detailed in Section 3.5. Coded as the price on the multiple price list immediately below the minimum price at which a subject opted to receive the corresponding cash reward rather than the flu shot coupon. If a subject opted to trade-in the coupon for cash at all prices, WTP will be coded as 0 . Because our funding is currently limited, we may not have the power to detect all differences but we are actively pursuing sources.

\subsubsection{Beliefs about Flu Shot Safety}

Primary outcomes: ${ }^{22}$

- Flu shot safety: Collected before and after display of the video at baseline. Measured by answer to survey question "Take 100 adult men, selected from your community at random. Let's say all of the 100 adult men receive a flu shot at the start of the flu season. How many of them do you believe get the flu from the flu shot?". Coded as 100 minus the subject's answer. ${ }^{23}$

- Flu shot safety: Collected before and after display of the video at baseline. Measured by answer to survey question "Take 100 adult men, selected from your community at random. Let's say all of the 100 adult men receive a flu shot at the start of the flu season. How many of them do you believe get the flu from the flu shot?". Coded as 100 minus the subject's answer.

\footnotetext{
${ }^{21}$ The index is based on baseline responses only to reduce any bias due to selective attrition between baseline and endline.

${ }^{22}$ Note we also considered incentivizing the belief elicitation, whereby people would make predictions and their guesses would be rewarded if correct. However we found it challenging to find an accepted arbitrator of the "truth".

${ }^{23}$ We have developed a novel interactive graphic for this and the question above that use a frequentist interpretation of probability and involves highlighting gray individual stick men with blue shading. This form of probability has been found to be easier to understand than others - see work by Gerd Gigerenzer.
} 
- Self-Confidence on Flu Shot Safety: Collected before and after display of the video at baseline. Measured by answer to survey question that asks subject to distribute 10 balls over 10 different bins. Each bin represents a range of people, out of 100, who get sick from the flu shot. Coded as total number of balls that are put into the "0-9" bin. ${ }^{24}$

Index of beliefs - primary:

- Includes all variables from Section 5.1.3.

Secondary outcomes: $:^{25}$

- Flu shot safety: Collected at endline. Answer to the survey question phrased exactly as the question described in "Flu shot safety".

Index of beliefs - secondary:

- Includes all variables from the "Secondary outcomes" section of Section 5.1.3.

\subsubsection{Social Distance Between Sender and Receiver}

All of the outcomes listed will be collected at baseline.

- Measure of Social Distance 1: Collected after display of the video at baseline. Measured by answer to the survey question "If a person like the one in the video was located near you, would you want to ask him about other health issues?" - coded as 1 if yes, and 0 if no.

- Measure of Social Distance 2: Collected after display of the video at baseline. Measured by answer to the survey question "Would a person like the one in the video be a contact in your phone or a friend on social media?" - coded as 1 if yes, and 0 if no.

\subsubsection{Sender and Signal Rating}

All of the outcomes listed will be collected at baseline.

- Likelihood of recommending the video to friends or family - self: Collected after display of the video at baseline. Measured by answer to the survey question "How likely are you to recommend this video to your friends or family? - scaled from 0 (Not at all likely) to 10 (Extremely likely).

- Trustworthiness: Answer to survey question "How much do you agree or disagree with the following statement?: I trust the person in the video to give me medical advice. - Disagree strongly/Disagree/Neither agree nor disagree/Agree/Agree strongly". Coded as -2, -1, 0, 1, 2.

- Qualification: Answer to survey question "How much do you agree or disagree with the following statement?: The person in the video is qualified to give me medical advice. - Disagree strongly/Disagree/Neither agree nor disagree/Agree/Agree strongly". Coded as -2, -1, 0, 1, 2.

\footnotetext{
${ }^{24}$ We have developed a new graphic interface for this question based on work by Delavande and Rohwedder (2008). See a picture of the graphic in the Appendix.

${ }^{25}$ All of the outcomes listed will be collected at endline
} 
- Applicability: Answer to survey question "How much do you agree or disagree with the following statement?: The information provided in the video applies to people like me. - Disagree strongly/Disagree/Neither agree nor disagree/Agree/Agree strongly". Coded as -2, -1, 0, 1, 2.

For the outcomes listed, a subject will be assigned into one of the ten actors' picture. All of the outcomes listed will be collected at endline.

- Subjective Rating - Age: "How old do you think this person is?"

- Subjective Rating - Education: "What is the highest degree or level of schooling that you think the person completed? - 1 (Less than a high school diploma), 2 (High school diploma or equivalent), 3 (Some college but no degree), 4 (Associate's degree), 5 (Bachelor's degree), and 6 (Graduate degree)"

- Subjective Rating - Trustworthiness: "How trustworthy is this person? - scaled from 1 (Not at all Trustworthy) to 10 (Extremely Trustworthy)"

- Subjective Rating - Attractiveness ${ }^{26}$ : "Please pick the three most attractive individuals from the list below."

Index of messenger rating:

- Includes all variables collected at baseline from the Section 5.1.5.

\subsubsection{Attention / Recall}

Primary outcomes: ${ }^{27}$

- Frequency recall: Measured by answer to the survey question What did the person in the video say about who should get the flu shot? - Everyone 6 months and older/Everyone 5 years and older/Everyone 18 years and older/I don't know. Coded 1 if selected "Everyone 6 months and older", and 0 otherwise.

- Flu shot ingredient recall: Measured by answer to the survey question What did the person in the video say about what the flu shot contains? - Contains active flu virus/Contains no active flu virus/I don't know. Coded 1 if selected "Contains no active flu virus", and 0 otherwise.

- Background color recall: This question is incentivized: Subjects will earn an extra $\$$ if they answer correctly. Measured by answer to the survey question What color was background displayed in the video? - Gray/White/Blue/I don't know. Coded 1 if selected "Blue", and 0 otherwise.

Index of attention/recall - primary:

- Includes all variables from the Section 5.1.6.

Secondary outcomes: $:^{28}$

\footnotetext{
${ }^{26}$ A subject will be given the pictures of 10 actors.

${ }^{27} \mathrm{All}$ of the outcomes listed will be collected at baseline.

${ }^{28} \mathrm{All}$ of the outcomes listed will be collected at endline.
} 
- Gender recall: Measured by answer to the survey question Was the person in the video a man or a woman? - Man/Woman/I don't know. Coded 1 if "Man", and 0 otherwise.

- Race recall: Measured by answer to the survey question What was the race of the person in the video? - Black/White/Other/I don't know. Coded 1 if selected correct answer, and 0 otherwise.

- Expert recall: Measured by answer to the survey question What was the person in the video wearing? - A doctor's coat/Casual clothes/I don't know. Coded 1 if selected correct answer, and 0 otherwise.

Index of attention/recall - secondary:

- Includes all variables from the "Secondary outcomes" section of Section 5.1.6.

\subsubsection{Beliefs about Coronavirus Infection}

All of the outcomes listed will be collected at endline.

- Likelihood of getting the coronavirus infection - self: How likely do you think you are to get the coronavirus infection? - scaled from 0 (Not at all likely) to 10 (Extremely likely).

- Likelihood of getting the coronavirus infection - self: If there was a coronavirus vaccine available to you, how likely would you be to get the vaccine? - scaled from 0 (Not at all likely) to 10 (Extremely likely).

\subsection{Index Construction}

We plan to construct indices of all outcomes related to beliefs in the flu vaccine, WTP, likelihood of getting a flu shot, and coupon redemption according to the procedure outlined in Anderson et al. (2008) and Kling Liebman and Katz (2007). We will also create additional indices of other outcomes related to beliefs about the actor in the video and attention to the video. XXYY decide on groupings

\subsection{Balance Checks}

We will conduct a series of balance tests across treatment arms to ensure that there are no chance differences between subjects in the various arms. We will regress characteristics on indicators for the arms and test their individual and joint significance. Balance tests will be conducted using the following subject background covariates: ${ }^{29}$

- Age

- Income

- Married

\footnotetext{
${ }^{29}$ We will also have a balance test for the actors across race. Caucasian respondents are included reducing the chance that a racial concordance effect for African-American subjects, for example, is being driven by the African-American actors being more attractive, higher quality etc than the Caucasian actors. This is because the same set of African-American actors/videos would not have the same predicted effect on Caucasian subjects. It's also possible that if there are differential perceptions across expert race by respondent race, this is mediating any racial concordance effect we find.
} 
- Living in Urban

- High School Completion

- Insured

- Subjective Health Status

- Prior Subjective Belief on Cost of Flu Shot

- Ever Vaccinated in the Past 5 Years

- Has PCP

- Prior Belief in Community Flu shot Take-up

- Prior Belief on Flu Effectiveness and Safety

\subsection{Treatment Effects}

We intend to measure the treatment effects in the following primary outcomes:

\subsubsection{Beliefs}

- $y_{1}$ : difference between prior and posterior beliefs about flu shot safety, such that:

$$
y_{1 i}=\mu_{1 i}^{0}-\mu_{1 i}^{1}
$$

where $\mu_{1 i}^{0}$ is subject $i^{\prime}$ s prior belief on the perceived safety of the flu vaccine, while $\mu_{1 i}^{1}$ is subject $i^{\prime}$ s update belief on the perceived safety. If the information from the video shifts the subject's priors to the truth, $y_{1 i}$ is positive.

- $y_{2}:$ difference between prior and posterior intention for a flu shot, such that:

$$
y_{2 i}=\mu_{2 i}^{1}-\mu_{2 i}^{0}
$$

where $\mu_{2 i}^{0}$ is subject $i^{\prime}$ s prior intention for getting a flu shot, while $\mu_{2 i}^{1}$ is subject $i^{\prime}$ s posterior intention for getting a flu shot. If the subject becomes more willing to get a flu shot after watching the video, $y_{2 i}$ is positive.

\subsubsection{Actions}

- $y_{3}$ : highest WTP price for a flu shot coupon within a set of prices, $\$ 1, \$ 2, \$ 5, \$ 10$. If a subject rejects all the prices, it is recorded as $\$ 0$.

- $y_{4}$ : pharmacy look-up, which is equal to 1 if the subject wanted a link to look up pharmacy where he can redeem his coupon, or 0 otherwise.

- $y_{5}$ : coupon redemption, which is equal to 1 if redeemed, or 0 otherwise. 


\subsubsection{Endorse}

- $y_{6}$ : likelihood to recommend the video to friends or family.

- $y_{7}$ : likelihood to recommend a flu shot to friends or family after watching the video.

\subsubsection{Ratings}

- $y_{8}:$ video rating about trustworthiness of the actor in providing medical advice (scaled from 1 to $5)$.

- $y_{9}$ : video rating about qualification of the actor in providing medical advice. (scaled from 1 to 5 )

\subsubsection{Social Proximity}

- $y_{10}$ : willingness to ask the actor about other health issues, which is equal to 1 if willing, or 0 otherwise.

- $y_{11}$ : likelihood of having the one in the video in the subject's contact, which is equal to 1 if willing, or 0 otherwise.

Using these variables, we construct six indices, for each individual $i$, such that:

- $Y_{i, \text { Beliefs }}^{\prime}$ consisted of $y_{1}$ and $y_{2}$

- $Y_{i, \text { Actions }}^{\prime}$ consisted of $y_{3}, y_{4}$ and $y_{5}$

- $Y_{i, \text { Endorse }}^{\prime}$ consisted of $y_{6}$ and $y_{7}$

- $Y_{i, \text { Ratings }}^{\prime}$ consisted of $y_{8}$ and $y_{9}$

- $Y_{i, \text { Soc.Prox. }}^{\prime}$ consisted of $y_{10}$ and $y_{11}$

- $Y_{i, A l l}^{\prime}$ consisted of $y_{1} \ldots y_{11}$

We intend to test the following hypotheses with this outcome variable:

H1(a): $Y^{\prime}$ is higher for subjects randomized to a race-concordant expert than those randomized to a race-discordant expert.

$$
Y_{i, j}^{\prime}=\alpha+\beta_{1} \mathbb{1}_{i}^{c_{1} \mid a=1, e=0}+X_{i}^{\prime} \Omega+\epsilon_{i}
$$

where $j$ is a type of indices among Beliefs, Actions, Endorse, Ratings, Soc. Prox. or All (as described abov), and $i$ is an individual subject. The following indicators describe subjects' treatment status:

- $c_{1}$ indicates whether a respondent is assigned to a race-concordant sender; 1 if so, or 0 otherwise.

- $a$ indicates whether a respondent is assigned to an expert sender; 1 if so, or 0 otherwise. 
- $e$ indicates whether a respondent is assigned to receive an empathetic message; 1 if so, or 0 otherwise.

$\mathbb{1}_{i}^{c_{1} \mid a=1, e=0}$ is an indicator for random assignment to a race-concordant setting for the respondents assigned to an expert sender delivering no empathetic message.

$X$ is a vector of attributes of the subjects and of the actors they are assigned to. We will obtain perceptions of the actors from a separate sample of men that are similar to the target population. ${ }^{30}$ Ideally these subject and actor characteristics would be balanced across arms but it's possible there is imbalance by chance. Additional important controls in our context may be the timing in which recruitment occurs relative to the flu season and the local intensity of the flu virus nationally or at a more local geographic level (i.e date and place of recruitment). We will use double-selection LASSO to choose a control set. ${ }^{31}$

The coefficients of interest are the $\beta$. If race concordant pairs update more than race-discordant among those assigned to an expert sender delivering no empathetic message, then $\beta_{1}$ should be positive (negative) and significant for the outcomes of effectiveness (harm). ${ }^{32}$

H1(b): $Y^{\prime}$ is higher for subjects randomized to a gender-discordant expert than those randomized to a gender-concordant expert within race concordance treatment group.

$$
Y_{i, j}^{\prime}=\alpha+\beta_{2} \mathbb{1}_{i, r=c}^{c_{2} \mid a=1, e=0}+X_{i}^{\prime} \Omega+\epsilon_{i}
$$

where $c_{2}$ indicates whether a respondent is assigned to a gender-concordant sender; 1 if so, or 0 otherwise. $\mathbb{1}_{i, r=c}^{c_{2} \mid a=1, e=0}$ is an indicator for random assignment to a gender-concordant expert actor among the respondents assigned to a racial concordant sender delivering a standard message, M1. If $\beta_{2}$ is negative and significant, this suggests that a subject updates more with gender-discordant expert in a racial concordant setting.

\section{H2: Racial concordance is more important for Blacks than for whites.}

$$
Y_{i, j}^{\prime}=\alpha+\beta_{3} \mathbb{1}_{i}^{c \mid a=1, e=0}+\beta_{4} \mathbb{1}_{i}^{r=b}+\beta_{5}\left[\mathbb{1}_{i}^{c \mid a=1, e=0} \cdot \mathbb{1}_{i}^{r=b}\right]+X_{i, r}^{\prime} \Omega+\epsilon_{i}
$$

where $\mathbb{1}_{i}^{c \mid a=1, e=0} \cdot \mathbb{1}_{i}^{r=b}$ is an interaction term between a racial concordance effect and subject's race. This term tests the effect of racial concordance(or discordance) across Black and white respondents.

\footnotetext{
${ }^{30} \mathrm{~A}$ subset of these questions are also asked in the baseline survey to the experimental sample. These variables include perceived age, qualifications, education, attractiveness, and trustworthiness. We will also use a voice analytic software to characterize the actors' voices.

${ }^{31}$ http:/ /www.nber.org/programs/dev/slides/18Duflo.pdf.

${ }^{32}$ This follows Hjort et al. (2019) who examine posterior beliefs based on the signal (re: early childhood intervention effect size) and the prior.
} 
H3: The racial concordance effect will be greater for a Black respondent assigned to a Black expert than for a Black respondent assigned to a Black layperson sender.

$$
Y_{i, j, r}^{\prime}=\alpha+\beta_{6} \mathbb{1}_{i, r=b}^{a \mid c=1, e=0}+X_{i, r=b}^{\prime} \Omega+\epsilon_{i, r=b}
$$

where $\mathbb{1}_{i, r=b}^{a \mid c=1, e=0}$ is an indicator for random assignment to an expert actor among the Black respondents assigned to a racial concordant sender delivering a standard message, M1. If $\beta_{4}$ is positive and significant, this suggests that an expert is specifically important for Black respondents in terms of updating beliefs in a racial concordant setting.

H4: For Black respondents, $Y^{\prime}$ is higher for subjects randomized to a race-discordant expert delivering an empathetic message, M2, than those randomized to a race-discordant expert delivering a standard message.

$$
Y_{i, j, r}^{\prime}=\alpha+\beta_{7} \mathbb{1}_{i, r=b}^{e \mid a=1, c=0}+X_{i, r=b}^{\prime} \Omega+\epsilon_{i, r=b}
$$

where $\mathbb{1}_{i, r=b}^{e \mid a=1, c=0}$ is an indicator for random assignment to receive a empathetic message among the Black respondents assigned to a race-discordant expert sender. If $\beta_{6}$ is positive and significant, this suggests that, in a race-discordant setting, validating perceived mistreatment in the past by an expert figure makes Black respondents update their beliefs more.

\subsection{Heterogeneous Effects}

Important secondary analyses will include investigating variation in the treatment response through interaction effects and sample splits. In particular, we are interested in whether the:

- persuasion treatment effects (TE) (racial and gender concordance, expert and/or empathy) are different for Black and white respondents

- persuasion treatment effects interact with each other

- TE vary by level of attention ${ }^{33}$

- TE vary by priors (beliefs about safety of shot)

- TE vary by perceived cost of flu shot

- TE vary by sociodemographic characteristics or medical history of the respondent (e.g. insurance status, marital status, has PCP, education)

- TE vary by "social distance" between the sender and receiver. ${ }^{34}$

- TE vary by local conditions, such as flu severity, flu shot availability, perceived and actual number of individuals vaccinated in community

- TE vary by distance to nearest pharmacy

- TE vary by double selection LASSO for finding heterogeneous treatment effects

\footnotetext{
${ }^{33}$ Attention may also be an outcome, if so we would be estimating complementary effects.

${ }^{34}$ Social distance proxied by age, education, race, income.
} 


\subsection{Standard Error Adjustments}

Although the level of randomization is the individual (respondent) there could be correlation across individuals randomized to the same actor-script-expert combination. Since we have 40 such treatment clusters we will plan to cluster at this level. We will also use randomization inference to calculate p-values (i.e. RI-test command developed by Simon Heß). ${ }^{35}$

\subsection{Attrition}

Attrition will be defined as those who have viewed the video but then drop out of the survey afterwards and do not complete it within four days of initiation. ${ }^{36}$ We will check that attrition is not differential across study arms. We will send respondents links to complete the survey from the point that they dropped out. ${ }^{37}$ The target for the sample is 3,350 completed surveys.

\section{COVID-19 Addendum}

In light of the relevance of vaccination take-up in addressing the COVID-19 pandemic, particularly among groups with historical mistrust of medical institutions, the main study will include an additional module designed to examine the effect of a concordant source on demand for information on a potential COVID-19 vaccine. This module will be presented to participants in the baseline survey following the influenza vaccination modules.

Subjects will be invited to receive the results of an upcoming expert review of a COVID-19 vaccine. The institution conducting the expert review will be randomized to either be the Food and Drug Administation (FDA) or the National Medical Association (NMA), the largest national organization representing African American physicians in the United States. The two messages presented to participants are reproduced below.

Message 1C (M1C) reads:

The National Medical Association (NMA), which represents Black physicians and health professionals in the US, will perform an independent review of the safety and efficacy of any COVID-19 vaccine approved by the FDA. Once a vaccine has been developed and the NMA has completed its review, would you like to receive an email notification with the results of the review?

Message 2C (M2C) reads:

The Food and Drug Administration (FDA) has formed an advisory committee of experts, who will perform an independent review of the safety and efficacy of any COVID-19 vaccine approved by the FDA. Once a vaccine

\footnotetext{
${ }^{35}$ See https://github.com/simonheb

${ }^{36}$ Qualtrics allows up to four days to finish the survey after starting it.

${ }^{37}$ This is a modification from the originally posted plan on 10/31/19 and takes into account that some subjects had technical difficulty returning to the survey after clicking on the map.
} 
has been developed and the advisory committee has completed its review, would you like to receive an email notification with the results of the review?

The outcomes of interest will be demand for information on COVID-19 vaccine safety and efficacy, as well as interest in learning more about personally participating in a COVID-19 vaccine trial. The former will be measured using the share of respondents demanding information on COVID-19 vaccine safety and efficacy from the assigned source. The latter will be measured using the share of respondents indicating their interest in learning about opportunities provided through the National Institutes of Health (NIH) to participate in COVID-19 vaccine trials. Heterogeneity by race will be examined.

\section{Research Team}

The principal investigators of this study are Marcella Alsan and Sarah Eichmeyer. They will contribute equally to all stages of the project - that is the design and execution of the field experiment, as well as the analysis of the collected data. Min Jeong (Joyce) Kim and Nikhil Shankar will support the project as research assistants, and will assist in all aspects of project implementation.

\section{Funding and Ethics}

Funding is provided by the J-PAL Health Care Delivery Initiative from MIT as well as Harvard Kennedy School. The IRB at Harvard is serving as the primary institution of record and has entered into a reliance agreement with Stanford and MIT.

\section{References}

[1] Allcott, Hunt, Luca Braghieri, Sarah Eichmeyer, and Matthew Gentzkow. 2019. "The Welfare Effects of Social Media." NBER Working Paper, 25514.

[2] Alsan, Marcella, Owen Garrick, and Grant Graziani. 2019. "Does Diversity Matter for Health? Experimental Evidence from Oakland." American Economic Review 109(12): 4071-4111.

[3] Anderson, Michael L. 2008. "Multiple inference and gender differences in the effects of early intervention: A reevaluation of the Abecedarian, Perry Preschool, and Early Training Projects." Journal of the American statistical Association 103(484): 1481-1495.

[4] Centers for Disease Control. 2017. Behavioral Risk Factor Surveillance System. Accessed September, 2019. https://www.cdc.gov/brfss/annual_data/annual_2017.html.

[5] Duflo, Esther. 2018. Machinistas meet randomistas: useful ML tools for empirical researchers. Accessed September, 2019. https://www. nber.org/programs/dev/slides/18Duflo.pdf.

[6] Heß, Simon. 2017. "Randomization inference with Stata: A guide and software." Stata Journal 17(3):630-651 
[7] Hjort, Jonas, Diana Moreira, Gautam Rao, and Juan Francisco Santini. 2019. "How Research Affects Policy: Experimental Evidence from 2,150 Brazilian Municipalities." NBER Working Paper, 25941.

[8] Kling, Jeffrey R., Jeffrey B. Liebman, and Lawrence F. Katz. 2007. "Experimental analysis of neighborhood effects." Econometrica 75(1): 83-119.

[9] Srinivas, Sindhu, Celeste Durnwald, Laura Line, Melissa Patti, Melissa Bucher, Shayna Cunningham, and Valerie Riis. 2019. "“Safe Start": A Community Health Worker Program That Improves Perinatal Outcomes in High Risk Women." American Journal of Obstetrics and Gynecology 20(1): 83119.

[10] Prochaska, James O., and Wayne F. Velicer. 1997. "The transtheoretical model of health behavior change" American journal of health promotion 12(1): 38-48. 


\section{Appendix}

\section{A. Baseline Survey Link}

https://harvard.az1.qualtrics.com/jfe/form/SV_ellmX4HMb5h3NVH

\section{B. Infomercial Video Link}

- Version Message 1: https://youtu . be/CxxWBT0ew-U

- Version Message 2: https : //youtu . be/TlruIaBOk3o

\section{Distribution of Beliefs}

This next question further helps us understand how certain you are of your answer regarding the side effects of the flu shot.

Again, consider the group of 100 adult men selected at random from your community, and suppose all of them get the flu shot.

You have 10 balls that you can put in 10 different bins, reflecting what you think are the chances out of 10 that the number of men who get sick from the flu shot falls in each bin. The more likely you think it is that the number of men with side effects falls in a given bin, the more balls you should place in that bin.

For example, if you put all the balls in one bin, it means you are certain the number of men that will fall sick from the flu shot is somewhere in that range.

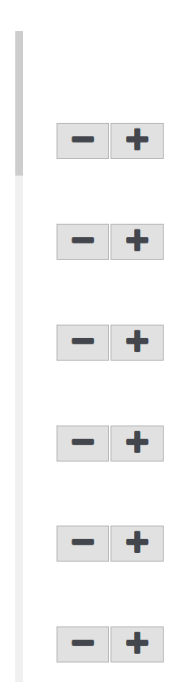

\section{Information on Initial Pilots}

Prior to launching the main survey, we ran two pilots in order to calibrate our questions and experimental design. Our first pilot included approximately 1000 male subjects recruited using the Qualtrics panel. Unfortunately, our targeting of low-education was not precise - 500 of the subjects had some college education. We only asked half the sample priors, and piloted 16 treatment arms, making it difficult to detect effects. However, among less educated, African-American subjects, there was some indication that the aforementioned interventions might prove effective (see power calculation above for further details).

During the first pilot, we had difficulty filling in cells for older African-American men using a Qualtrics panel. We modified recruitment for part of our second pilot by adding online recruitment via Google/ Facebook. Unfortunately, targeting was more imprecise using online advertisements, and our cost per 
completed response was extremely high. In a second Qualtrics pilot that we ran concurrently with the online ads, we found that tightening our targeting criteria proved successful in fielding a sample representative of our population of interest, motivating our scaling-up of our second Qualtrics pilot.

We also noted in our first pilot that despite shifting posteriors in the direction of more favorable views of the flu vaccine, flu shot coupon redemption was virtually zero. This finding indicates that among the Qualtrics survey sample population, even our most "persuasive" intervention did not shift beliefs enough so as to pass individuals' net benefit thresholds and trigger coupon redemption. Thus, we added in our second pilot a deadline to the coupon (currently within 5 days of receipt) for entry into a lottery among those who redeem in that time frame.

Third, in the first pilot, we randomly assigned half the sample to a survey that elicited prior beliefs about the flu and the flu shot and the other half to a survey that did not elicit priors. The rationale was that while priors are key heterogeneity dimensions of interest and while they increase the power of our analysis, we were worried that they would trigger a "doubling-down" effect or lead individuals to engage System II more often, thereby muting treatment effects in an undesirable (because not externally valid) way. However, our initial pilot revealed no such effects. Therefore, starting in the second pilot, we plan on asking these questions to everyone. 